\title{
REHABILITACIÓN ACÚSTICA DE AULAS EN LA FACULTAD DE FILOSOFÍA Y LETRAS DE VALLADOLID - ESPAÑA
}

\author{
(ACUSTIC REHABILITATION OF CLASSROOMS IN THE PHILOSOPHY FACULTY \\ OF VALLADOLID - SPAIN)
}

Julio González Suárez, Dr. en C. Fisicas Prof. ETSAV.

Francesc Daumal i Domènech, Dr. Arquitecto Prof. ETSAB.

José Ignacio Sánchez Rivera, Dr. en C. Fisicas Prof. ETSAV

Fecha de recepción: 7-1II-91

\section{RESUMEN}

Se expone a continuación el proceso seguido para el acondicionamiento acústico de las aulas denominadas Sala de Alumnos, P. Mariana y J. Zurita, de la Facultad de

Filosofía y Letras de Valladolid, donde se ha pasado de unos tiempos de reverberación iniciales comprendidos entre 3,5 y 1 segundos (para los 20 tercios de octava que van de 100 a $8000 \mathrm{~Hz}$ ) a unos finales entre los 2,8 y los 0,3 segundos, para dichas frecuencias.
SUMMARY

The process for the acustic arrangement of the classrooms called Pupil's Room, P. Mariano and J. Zurita of the Philosophy Faculty of Valladolid is presented.

The initial reverberation times, which were in the range 3,5-1 seconds, have been changed to the range $2,8-0,3$ seconds (for the 20 thirds of octave between 100 and $8.000 \mathrm{~Hz}$ ).

\section{INTRODUCCIÓN}

\section{Problemática de las salas}

Presentamos un trabajo que es fruto de un intenso trasvase de información, realizado a modo de "Feed-back", entre Barcelona y Valladolid.

Se trata del acondicionamiento acústico de unas aulas de la Facultad de Filosofía de la Universidad de Valladolid, y por ello, al ser estas aulas ya existentes, se encuadra de lleno en lo que denominamos campo de la Rehabilitación acústica. Nos encontramos pues con un campo de la acústica que trata con obra ya existente, que debe acomodarse a unas circunstancias proyectuales y constructivas muy especificas y diferenciadas de los otros campos de trabajo de la acústica.

En concreto, las 3 aulas a rehabilitar eran la Sala de Alumnos, Jerónimo Zurita y Padre Mariana. Dichas aulas se hallan ubicadas en el primer piso del Edificio de la Facultad. Presentan forma prismática, y sus plantas pueden verse grafiadas a escala en la figura 1.

En esta figura se representa, además, el mobiliario y los puntos donde se efectuó el muestreo. Las dimensiones, antes de la remodelación, eran:

Sala de alumnos: $\quad 5,87 \times 8,25 \times 5,07 \mathrm{~m} \quad$ volumen $=242 \mathrm{~m}^{3}$

Padre Mariana: $\quad 7,88 \times 8,10 \times 5,07 \mathrm{~m} \quad$ volumen $=324 \mathrm{~m}^{3}$

Jerónimo Zurita: $\quad(5,28) 6,27 \times 8,11 \times 5,07 \mathrm{~m} \quad$ volumen $=237 \mathrm{~m}^{3}$

Según comentarios de la Sra. Decana de Filosofía, el problema de estas aulas radicaba en que "se oía muy mal", hasta el punto de no ser hábiles para impartir la docencia, sobre todo en la Sala de alumnos. 

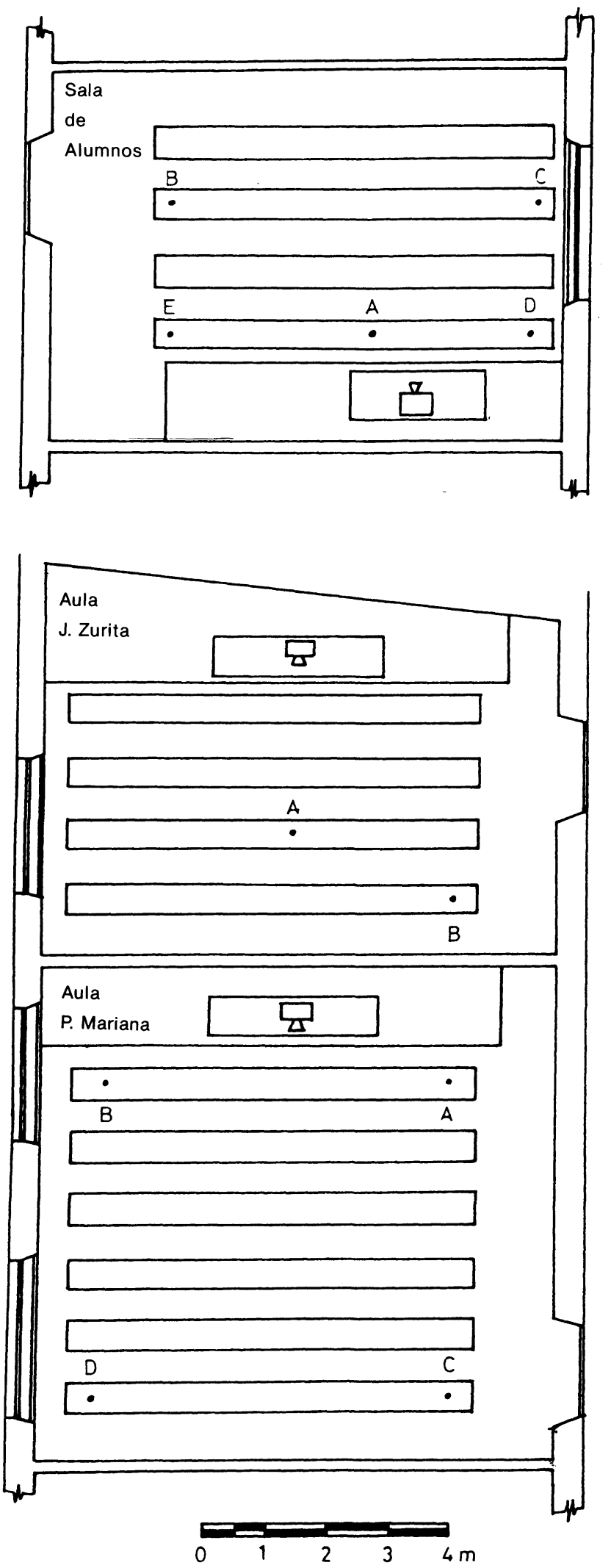

Fig. 1.-Planta de las aulas Sala de Alumnos, Jerónimo Zurita y Padre Mariana de la Facultad de Filosofía y Letras de la Universidad de Valladolid, con representación de su mobiliario, situación de la Fuente de Emisión Sonora y puntos de las aulas donde se tomaron medidas de Tiempo de Reverberación y Nivel Sonoro.
Por todo ello se decidió realizar unas mediciones de Tiempo de Reverberación y Nivel Sonoro, en los puntos de las aulas que se indican en la figura 1.

En principio nuestro estudio se centró en la Sala de Alumnos con el fin de analizar con cierto detalle las causas que pudieran originar una audibilidad tan deficiente y, en base a ese análisis, proponer soluciones para mejorarla y estudiar, una vez realizada la obra, las discrepancias que previsiblemente se pudieran presentar entre los valores esperados en función de los cálculos realizados y los valores experimentales que se obtuvieran. Esto nos permitiría ajustar mejor la solución a adoptar para la rehabilitación de las otras aulas.

\section{MEDICIONES REALIZADAS}

A la vista de la problemática planteada se realizaron las medidas de las variables acústicas (Tr y niveles de sonido) y de las dimensiones geométricas, necesarias para realizar el estudio.

El equipo acústico empleado ha consistido en:

Fuente sonora: 4224 de Brüel \& Kjaer.

Analizador acústico de Edificios 4418 de Brüel \& Kjaer. Micrófono: 4449 de Brüel \& Kjaer.

Tabla 1

\begin{tabular}{|c|c|c|c|c|c|c|}
\hline \multirow[t]{2}{*}{ frec. } & \multicolumn{2}{|c|}{ S. Alumnos } & \multicolumn{2}{|c|}{ J. Zurita } & \multicolumn{2}{|c|}{ P. Mariana } \\
\hline & $\mathrm{N}$ & $\mathrm{Tr}$ & $\mathrm{N}$ & $\operatorname{Tr}$ & $\mathrm{N}$ & $\mathrm{Tr}$ \\
\hline 100 & 90,2 & 3,4 & 87,6 & 3,2 & 85,6 & 2,3 \\
\hline 125 & 95,6 & 3,2 & 93,7 & 2,2 & 91,8 & 2,4 \\
\hline 160 & 97,1 & 2,6 & 95,0 & 2,4 & 92,9 & 2,3 \\
\hline 200 & 97,5 & 2,4 & 94,8 & 2,4 & 92,2 & 2,3 \\
\hline 250 & 98,4 & 2,3 & 95,0 & 2,1 & 92,7 & 1,9 \\
\hline 315 & 98,8 & 2,5 & 97,2 & 2,3 & 95,6 & 1,8 \\
\hline 400 & 99,7 & 2,3 & 96,7 & 2,3 & 96,0 & 2,0 \\
\hline 500 & 99,0 & 2,4 & 99,2 & 2,2 & 95,9 & 2,0 \\
\hline 630 & 100,8 & 2,4 & 99,8 & 2,3 & 97,1 & 2,0 \\
\hline 800 & 98,6 & 2,4 & 96,8 & 2,2 & 94,2 & 2,1 \\
\hline $\begin{array}{l}1000 \\
1250\end{array}$ & $\begin{array}{l}97,1 \\
94.1\end{array}$ & $\begin{array}{l}2,4 \\
2,5\end{array}$ & 95,5 & 2,4 & 93,2 & 2,2 \\
\hline 1600 & 91,9 & 2,3 & 90,9 & $\begin{array}{l}2,4 \\
2,3\end{array}$ & $\begin{array}{l}80,5 \\
89,5\end{array}$ & 2,1 \\
\hline 2000 & 97,3 & 2,2 & 95,6 & 2,1 & 93,5 & 2,0 \\
\hline 2500 & 98,1 & 2,2 & 97,5 & 2,2 & 93,6 & 1,9 \\
\hline 3150 & 95,1 & 2,1 & 93,3 & 2,1 & 90,5 & 1,8 \\
\hline 4000 & 89,8 & 1,9 & 88,9 & 1,7 & 86,7 & 1,7 \\
\hline 5000 & 85,1 & 1,6 & 78,8 & 1,5 & 80,9 & 1,5 \\
\hline 6300 & 77,9 & 1,4 & 76,8 & 1,4 & 74,5 & 1,3 \\
\hline 8000 & 73,8 & 1,1 & 71,9 & 1,0 & 70,4 & 1,0 \\
\hline
\end{tabular}

Tiempos de Reverberación (s) y Niveles Sonoros (dB) medidos en las aulas Sala de Alumnos, J. Zurita y P. Mariana antes de la realización de obras de acondicionamiento en las mismas. 
$\operatorname{Trev}(\mathbf{s})$

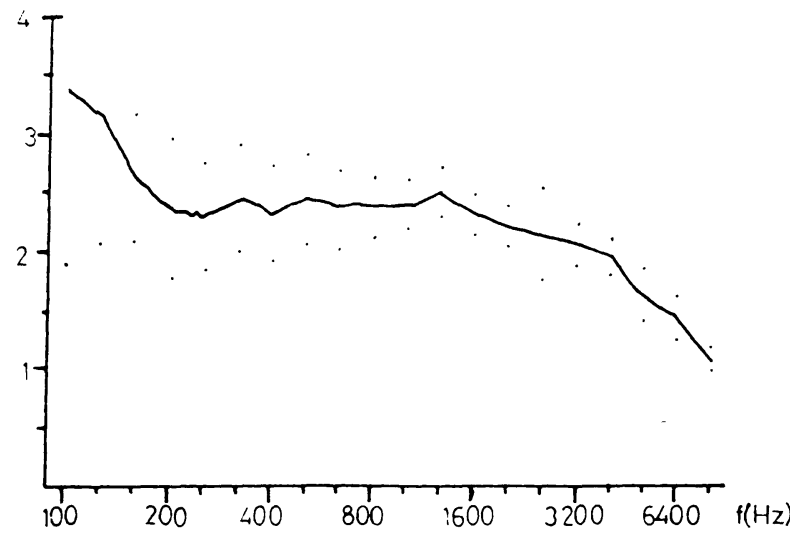

Fig. 2.-Representación de los Tiempos de Reverberación de Sala de Alumnos por tercios de octava, obtenidos como promedio de los 5 puntos de medida tomados en el aula (con sala vacía). Los puntos que rodean la linea continua indican las cotas de dispersión.

La colocación del equipo dentro de cada aula y las dimensiones de las mismas se indican en la figura 1.

Los resultados de los Tiempos de Reverberación, Tr y del Nivel Sonoro, N, se dan en la tabla I, para intervalos de frecuencias en tercios de octava.

Los valores de Tr de la tabla son la media aritmética de tres medidas realizadas en el mismo punto. En las figuras los valores se acompañan de una cota de dispersión $(\Delta)$, calculada como la semidiferencia entre el valor máximo y el mínimo en cada punto. Para el caso del nivel, se realizó una única medida en cada punto, promediando directamente el Analizador durante $5 \mathrm{se}$ gundos.

Téngase en cuenta que los Tr medidos (que figuran en la tabla l), son tiempos con sala vacía. Posteriormente se expondrán los valores obtenidos a partir de los cálculos realizados para obtener el Tr óptimo en sala llena.

El estudio de acondicionamiento se realizó fundamentalmente por la Sala de alumnos según se comentó anteriormente, siendo adoptada la misma solución por parte del Servicio Técnico de la Universidad para las aulas P. Mariana y J. Zurita. En la gráfica de la figura 2 se representan los tiempos de reverberación de Sala de Alumnos, obtenidos como promedio de los valores de los 5 puntos de medida (todo ello con sala vacía). Los puntillos que rodean a la línea continua indican las cotas de la semidiferencia entre el valor máximo y el mínimo para cada frecuencia.

\section{PROYECTO DE REHABILITACIÓN ACÚSTICA}

\section{Primeras impresiones de la Sala de Alumnos}

Las primeras impresiones que podriamos deducir a partir de los datos representados en la figura 2 y $\sin$ entrar en detalles eran que:

a) El problema debe estar centrado en la existencia de mucha reverberación, sobre todo para las frecuencias bajas y medias. Las dimensiones del aula son algo raras (especialmente en su altura) tal y como se deduce de las dimensiones geométricas.

$\operatorname{Trev}(\mathrm{s})$
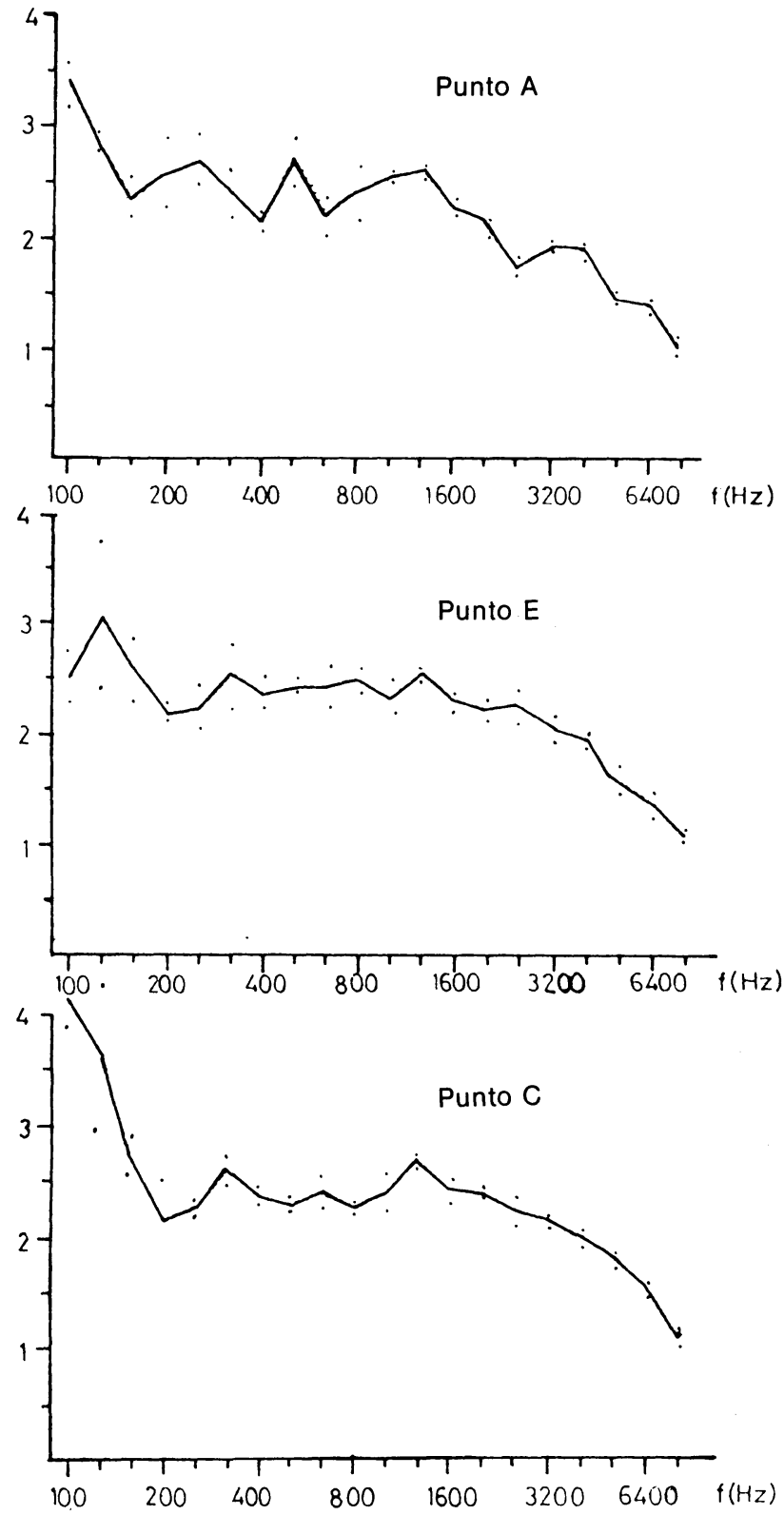

Fig. 3.-Representación de los Tiempos de Reverberación de Sala de Alumnos por tercios de octava, obtenidos para 3 puntos de medida tomados en el aula (con sala vacía). Los puntos que rodean a la línea continua indican las cotas de dispersión. 
b) Si tomamos como referencia la banda de frecuen. cia centrada en $500 \mathrm{~Hz}$, vemos que el tiempo de reverberación medio en el punto A es de 2,66 s. en sala vacia (véase figura 3 ), que según los cálculos previos se convierte en $1,15 \mathrm{~s}$. aproximadamente en sala llena.

Aceptando como primera aproximación la conveniencia de la ecuación:

$$
\mathrm{T}_{0}=\gamma \mathrm{V}(1 / 3)
$$

para el cálculo del tiempo de reverberación óptimo, y aceptando el valor de $\gamma=0,075$, resulta un $\mathrm{T}_{0}=0,47 \mathrm{~s}$.

c) Por otro lado, tomando como criterio la velocidad de amortiguamiento (Tesis doctoral F. Daumal), y bajo la hipótesis de que toda la superficie estuviera ocupada, resulta de 4,3 m/s.

d) A partir de cualquiera de los dos criterios mencionados, se observa que para este aula los valores se encuentran bastante alejados de los que pueden considerarse como óptimos.

e) Con la idea de mejorar la calidad acústica de la sala parece, en principio, que sería conveniente disminuir el tiempo de reverberación. Para conseguirlo, teniendo en cuenta la gran diferencia que existe entre los $T r$ de frecuencias graves y agudas, parece aconsejable colocar absorbentes selectivos, es decir, que incidan más sobre las frecuencias graves que sobre las agudas.

f) En base a esta propuesta una posible solución iría en el sentido de revestir las paredes hasta una altura de $1,5 \mathrm{~m}$ aproximadamente con una material poroso y un sistema de absorción mediante resonadores en el techo, buscando la absorción fundamentalmente para frecuencias graves. El sistema de resonadores que parecía más indicado (ver figura 4) consistía en planchas perforadas por distintos diámetros y colocadas a distintas distancias del techo (también para favorecer la difusión).

\section{Evaluación posterior}

Tras realizar un análisis más exhaustivo y riguroso del problema se entendió:

a) Que siendo un aula pequeña (sobre los $250 \mathrm{~m}^{3}$ ), los problemas de reverberación existentes pueden corregirse sin tener que recurrir a variaciones importantes de su volumetria (por otro lado limitadas por la presencia de ventanales y puertas de gran altura, o sofisticadas instalaciones difusoras que encarecen extremadamente la obra).

b) Que claramente se presentan las frecuencias propias del local (en las gráficas del Tr punto a punto de la figura 3), en coincidencia con la fórmula de Rayleigh, si bien en el margen de frecuencias estudiado, a partir del tercio de octava de $100 \mathrm{~Hz}$ su distanciamiento ya es bastante aceptable puesto que las proporciones del aula siguen un prisma unitario de 1,65/1,17/1. Ello deja al local bastante próximo de las proporciones óptimas de Bolt.
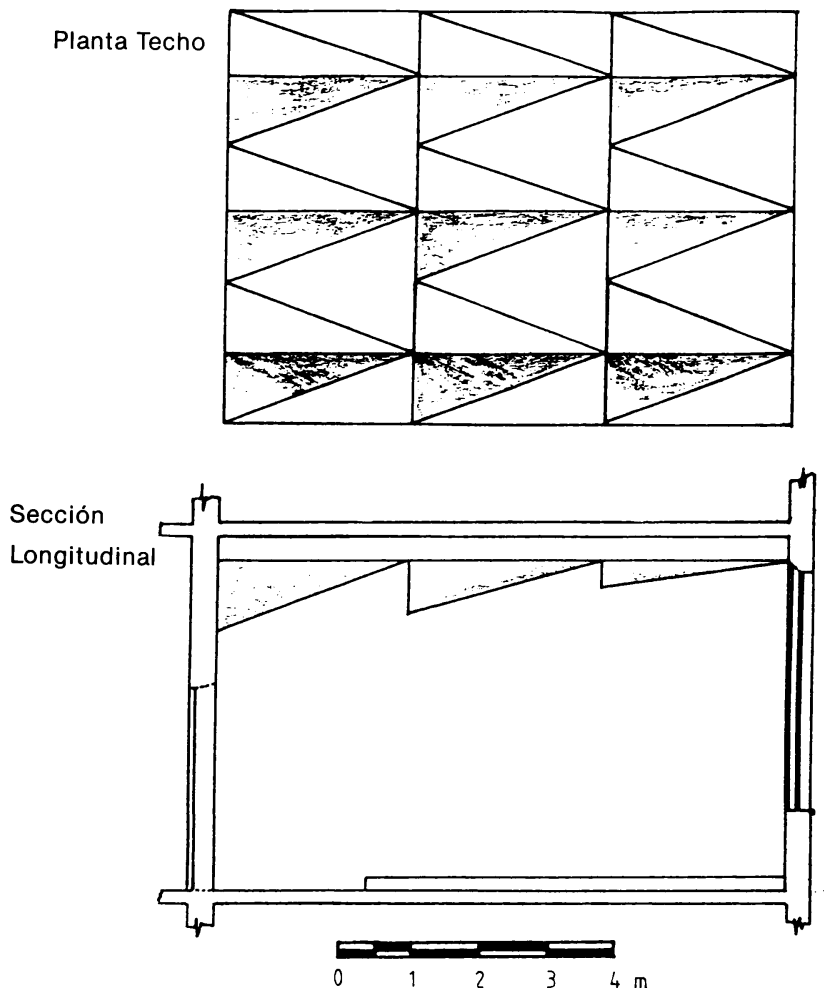

OPCION A

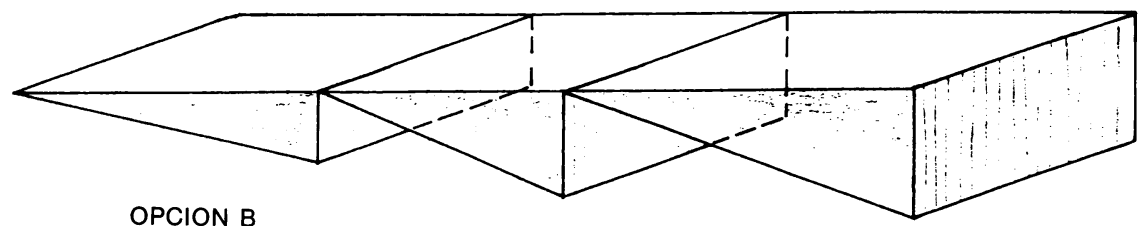

Fig. 4-Esquema de la primera propuesta de acondicionamiento para la Sala de Alumnos. Los paneles acústicos podrian ser de dos modelos (opción A y opción B). La opción $B$ se representa en su colocación sobre la planta del techo.

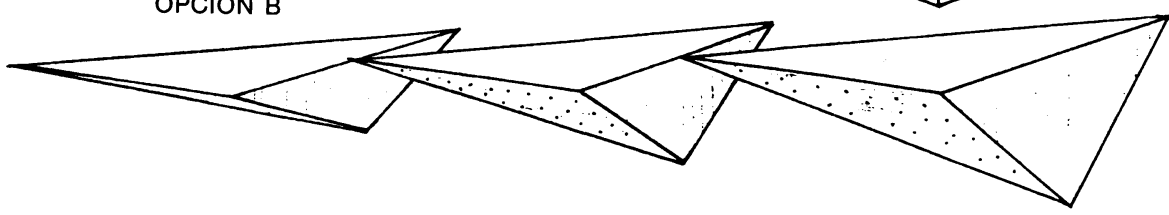

(c) Consejo Superior de Investigaciones Científicas

Licencia Creative Commons 3.0 España (by-nc) 
c) Que una reducción de la altura, por interposición de falso techo (dejando la altura en $4,70 \mathrm{~m}$ ), hará coincidir las nuevas proporciones $(1,75 / 1,24 / 1)$ con el límite de Bolt, optimizando la solución. Por otra parte, ello hará disminuir la elevada esponjosidad actual (5,9 $\mathrm{m}^{3}$ /ocupante).

d) Que el campo reverberante puede considerarse perfectamente difuso a partir de $250 \mathrm{~Hz}$, observándose cierto aumento del Tr en las bajas frecuencias al alejarse de la fuente sonora. En estos puntos alejados el Tr adopta valores muy elevados y los rayos directos llegan con menor intensidad, provocando todo ello una disminución importante del índice de intelegibilidad (ya escaso en el resto del aula).

e) Que una posible solución, tendente a igualar el Tr en todos los puntos del aula, consiste en eliminar la formación de las primeras frecuencias propias (axiles), mediante material absorbente en al menos uno de cada dos planos paralelos, la de las tangenciales mediante absorbente ubicado en al menos uno de los ángulos ortogonales y la de las oblicuas con absorbente dispuesto en al menos uno de los triedros. Como los modos tangenciales y oblicuos son superiores a los axiles, la solución debe tender a generar absorción en los planos contiguos entre sí. No se cree necesario el uso de los volúmenes difusores de la solución inicial por las razones antes expresadas.

$$
\begin{aligned}
& \text { N. de modos axiles: } \mathrm{N}_{\mathrm{a}}=11 \Delta \mathrm{f} / \mathrm{f} \\
& \text { N.o de modos tangenciales: } \mathrm{N}_{\mathrm{t}}=53 \Delta \mathrm{f} / \mathrm{f} \\
& \text { N. de modos oblicuos: } \mathrm{N}_{0}=47 \Delta \mathrm{f} / \mathrm{f}
\end{aligned}
$$

Donde $\Delta f / f$ representa la diferencia entre las frecuencias superior e inferior del intervalo escogido dividida por la frecuencia central del intervalo.

i) Que en base a los siguientes tiempos de reverberación óptimos, según Bossut:

$\begin{array}{llllllll}\frac{125}{1,25} & \frac{250}{1,00} & \frac{500}{0,75} & \frac{1 \mathrm{~K}}{0,75} & \frac{2 \mathrm{~K}}{0,75} & \frac{4 \mathrm{~K}}{0,65} & \frac{8 \mathrm{~K}}{0,60} & \frac{\mathrm{Hz}}{\mathrm{s}}\end{array}$

y aplicando en primera aproximación la fórmula de Sabine, corrigiendo los resultados por la absorción de ocupantes, por el mayor valor de la absorción del material corrector y por el ángulo de la primera incidencia del rayo sonoro, se obtienen:

- Absorbente frec. graves (125 a $250 \mathrm{~Hz}$ ) en techo de $36 \mathrm{~m}^{2}$ de superficie (efectividad por ubicación $50 \%$ ):

$$
\begin{aligned}
& \alpha_{125}=0,54 \\
& \alpha_{250}=0,60
\end{aligned}
$$

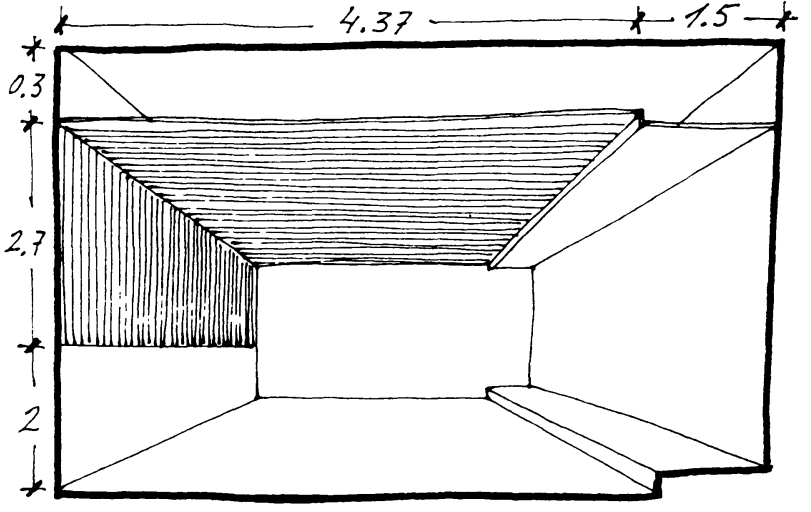

Fig. 5.-Esquema definitivo propuesto para la remodelación del aula Sala de Alumnos.

- Absorbente Frec. medias y aguas en techo y pared de $58 \mathrm{~m}^{2}$ de superficie (efectividad por ubicación $60 \%$ ):

$$
\begin{aligned}
\alpha_{500} & =0,57 \\
\alpha_{1000} & =0,52 \\
\alpha_{2000} & =0,47 \\
\alpha_{4000} & =0,61 \\
\alpha_{8000} & =0,42
\end{aligned}
$$

Por lo anteriormente especificado, un material idóneo para la corrección consistió en aglomerado de fibra de madera adherido con magnesita, suspendido en falso techo y adherido en las partes altas de las paredes. En las partes bajas de las paredes (hasta 1,50 metros del suelo) se optó por prever un arrimadero de madera no considerado absorbente al sonido.

Se recomentó un modelo de acabado tipo travertino, de $25 \mathrm{~mm}$ espesor y de $15 \mathrm{~kg} / \mathrm{m}^{2}$ dispuesto en falso techo y con manta de lana de roca en su parte superior, garantizando con ello plenamente la corrección.

Se creyó oportuno considerar que no todo el techo fuera absorbente, sino que sobre la tarima del estrado existiera un panel de yeso reflector (se puede ver un esquema del mismo en la figura 5).

En cualquier caso, se pensó necesario exigir el certificado de incombustibilidad de la solución, con grados admisibles tipos M1 (no inflamable) y M0 (incombustible).

\section{REALIZACIÓN DEL PROYECTO}

\section{Variables introducidas}

A la hora de ejecutar el proyecto que les presentamos, el Equipo Técnico de la Universidad que realizó la obra consideró, por una parte, que las planchas propuestas para el falso techo eran demasiado pesadas y que exis- 
Coef. de absorción

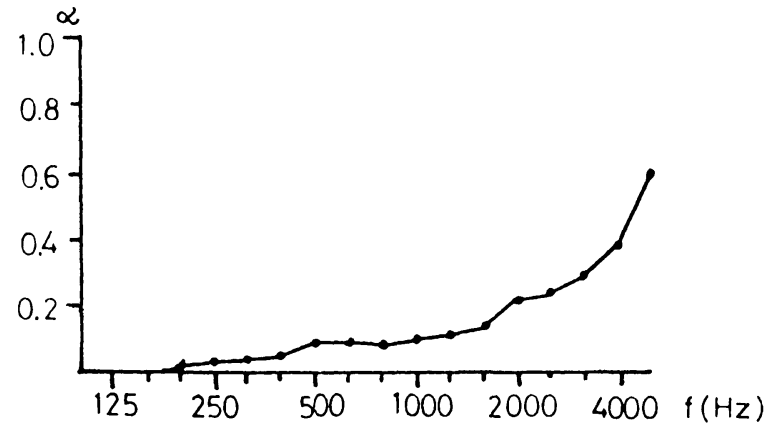

Fig. 6.-Representación gráfica del Coeficiente de Absorción por tercios de octava, $\alpha$, de la pintura tipo GGP -579 .

Coef. de absorción

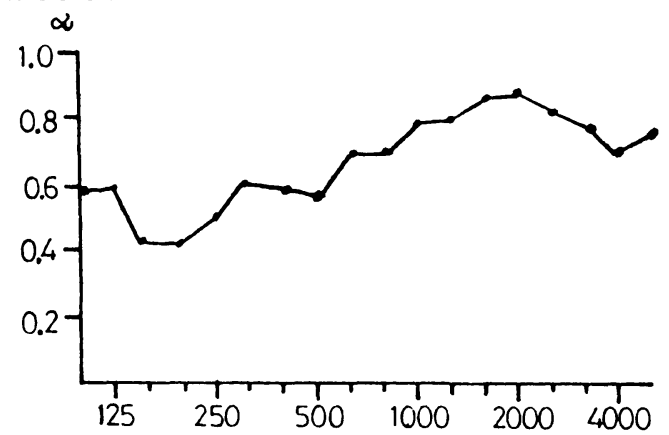

$\mathrm{f}(\mathrm{Hz})$

Fig. 7.-Representación gráfica del Coeficiente de Absorción por tercios de octava, $\alpha$, de los paneles SONEBEL FM12 con perforación irregular.

tía cierto riesgo para los ocupantes de las aulas y, por otra, que a su juicio era suficiente con acomodar el techo, prescindiendo del panel de yeso reflector, y sustituir los absorbentes en los cerramientos verticales por una pintura tipo GGP - 579 de la que exponemos el coeficiente de absorción en la figura 6 .

Nuestras sugerencias, tanto en lo relativo a la colocación del faso techo como en lo previsto para colocar encima del estrado, y el que la pintura pudiera ser perjudicial -ya que la absorción se centra fundamentalmente en las frecuencias agudas - no fueron atendidas, instalándose en su lugar un falso techo mediante chapas de paneles SONEBEL FM12 con perforación irregular a 4,70 m del suelo, cuyas características de absorción se adjuntan en la figura 7 .

\section{MEDIDAS FINALES}

Una vez acondicionada la Sala de Alumnos se realizaron nuevamente medidas de Tr y Niveles con sala vacía, obteniendo los resultados que se exponen en la figura 8. En dichas gráficas, con el ánimo de visualizar mejor la diferencia entre el estado del aula antes y después de la remodelación, se exponen, además,
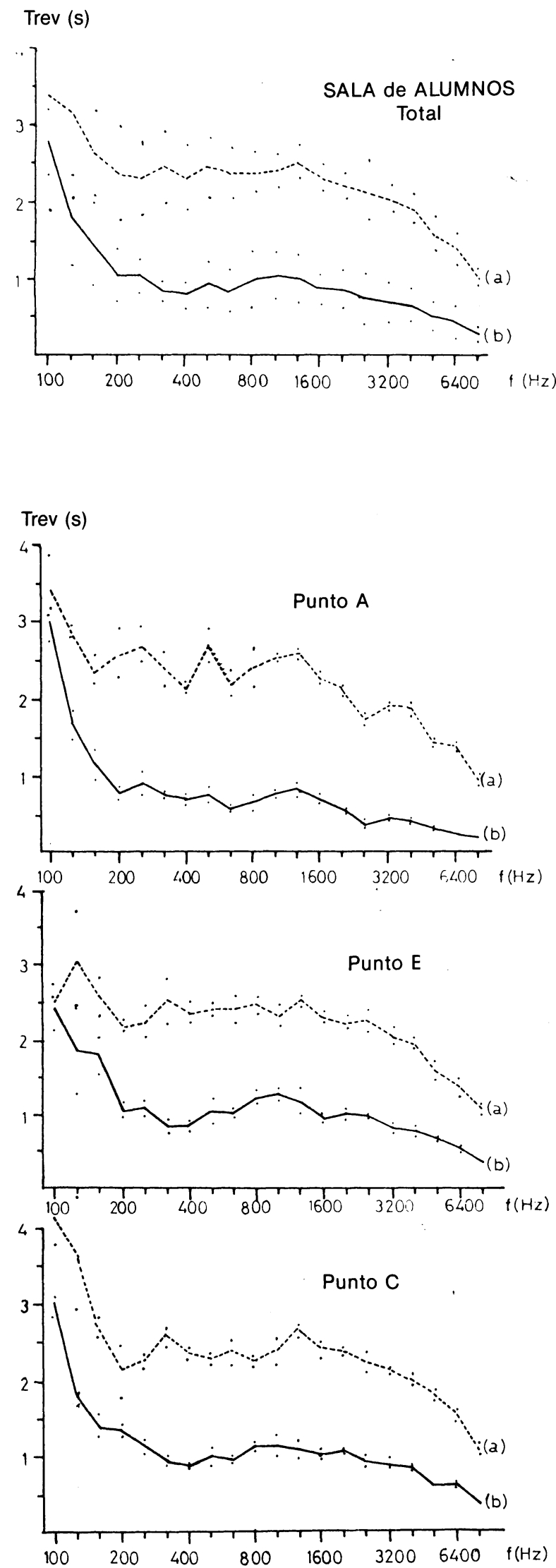

Fig. 8.-Representación de los Tiempos de Reverberación de la Sala de Alumnos por tercios de octava, obtenidos para toda la sala y para los 3 puntos indicados (con sala vacia) después del acondicionamiento (línea b) y antes del mismo (linea a). Los puntos que rodean las líneas indican las cotas de dispersión. 
Tabla II

\begin{tabular}{|c|c|c|c|c|c|c|}
\hline \multirow[t]{2}{*}{ frec. } & \multicolumn{2}{|c|}{ S. Alumnos } & \multicolumn{2}{|c|}{ J. Zurita } & \multicolumn{2}{|c|}{ P. Mariana } \\
\hline & $\mathrm{N}$ & $\mathrm{Tr}$ & $N$ & $\mathrm{Tr}$ & $N$ & $\operatorname{Tr}$ \\
\hline 100 & 86,8 & 2,8 & 85,9 & 2,1 & 84,6 & 1,8 \\
\hline 125 & 90,2 & 1,8 & 89,0 & 1,6 & 86,1 & 1,8 \\
\hline 160 & 89,6 & 1,4 & 90,4 & 1,0 & 88,2 & 1,1 \\
\hline 200 & 92,5 & 1,1 & 91,0 & 0,7 & 89,1 & 0,7 \\
\hline 250 & 93,9 & 1,0 & 90,5 & 0,7 & 88,5 & 0,6 \\
\hline 315 & 95,4 & 0,8 & 92,6 & 0,6 & 89,7 & 0,5 \\
\hline 400 & 93,6 & 0,8 & 92,6 & 0,6 & 89,9 & 0,6 \\
\hline 500 & 95,4 & 0,9 & 92,5 & 0,7 & 91,0 & 0,7 \\
\hline 630 & 97,6 & 0,8 & 95,0 & 0,7 & 91,1 & 0,8 \\
\hline 800 & 94,5 & 1,0 & 92,7 & 0,9 & 88,6 & 1,0 \\
\hline 1000 & 90,3 & 1,1 & 89,0 & 0,9 & 86,2 & 0,9 \\
\hline 1250 & 88,1 & 1,0 & 89,6 & 0,8 & 85,4 & 0,9 \\
\hline 1600 & 87,2 & 0,9 & 88,9 & 0,9 & 85,4 & 0,9 \\
\hline 2000 & 92,3 & 0,9 & 94,0 & 0,9 & 90,1 & 1,0 \\
\hline 2500 & 94,2 & 0,8 & 95,4 & 1,0 & 90,9 & 1,0 \\
\hline 3150 & 90,2 & 0,7 & 92,0 & 1,1 & 87,9 & 1,0 \\
\hline 4000 & 84,8 & 0,7 & 87,3 & 1,1 & 84,5 & 1,0 \\
\hline 5000 & 80,1 & 0,5 & 83,5 & 0,9 & 79,5 & 1,0 \\
\hline 6300 & 74,1 & 0,5 & 75,0 & 0,9 & 73,6 & 0,9 \\
\hline 8000 & 71,3 & 0,3 & 70,9 & 0,7 & 69,2 & 0,7 \\
\hline
\end{tabular}

Tiempos de Reverberación (s) y Niveles Sonoros (dB) medidos en la Sala de Alumnos, J. Zurita y P. Mariana después de la realización de obras de acondicionamiento en las mismas.

los valores medidos antes de la remodelación para los tres puntos especificados y el promedio global para la Sala de Alumnos. En la tabla ll exponemos también estos valores junto con los obtenidos en las otras dos aulas una vez hecha la remodelación por parte del Servicio Técnico de la Universidad, conforme a como se indicó anteriormente.

De igual forma, en los gráficos de la figura 9 se representan los Tr medios medidos en las aulas P. Mariana y J. Zurita antes y después de la remodelación, indicando cotas de dispersión de los valores experimentales.
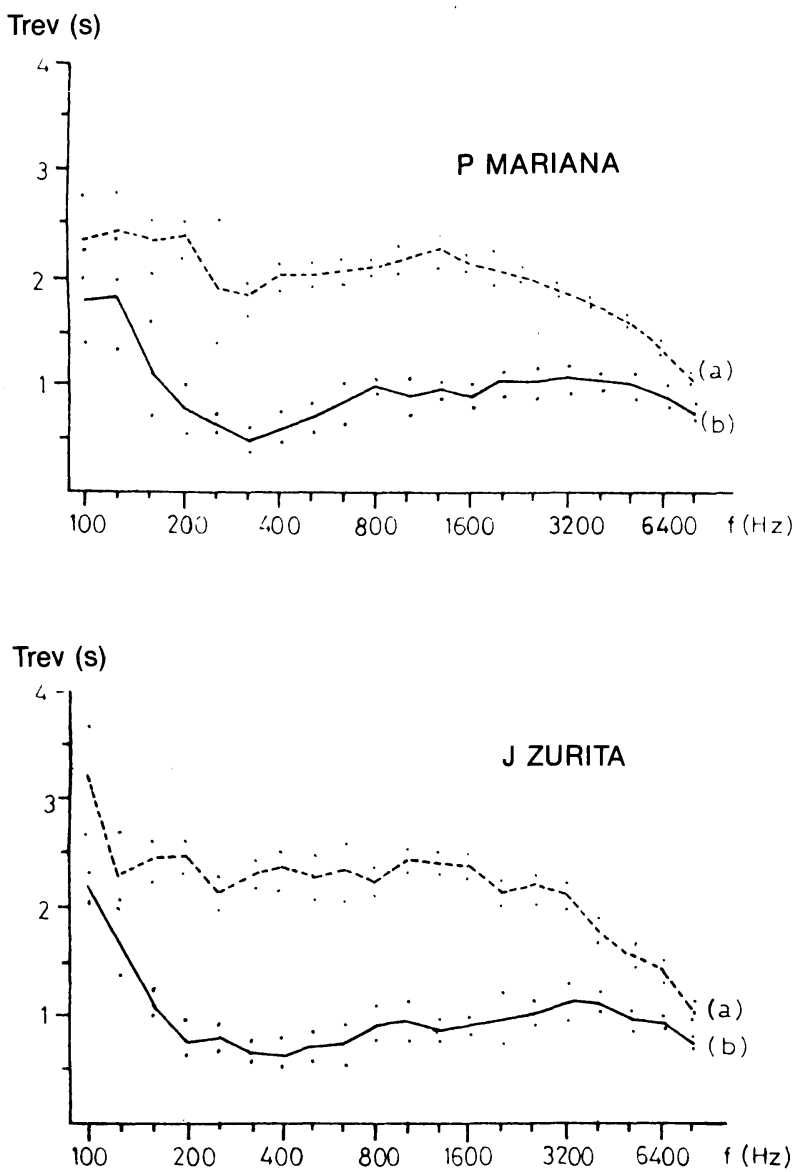

Fig. 9.-Representación de los Tiempos de Reverberación por tercios de octava de las aulas P. Mariana y J. Zurita obtenidos para toda la sala (con sala vacía) después del acondicionamiento (línea b) y antes del mismo (línea a). Los puntos que rodean las lineas indican las cotas de dispersión.

Conviene destacar que si bien de esta forma se han reducido sustancialmente los Tiempos de Reverberación, sin embargo los valores de los Niveles no han sufrido una modificación importante ni en su valor ni en su variación respecto de la frecuencia, como puede comprobarse comparando los valores de las tablas I y II.

\section{CONCLUSIONES}

A título de conclusiones, entendemos que la remodelación realizada ha mejorado apreciablemente las condiciones acústicas de las aulas, según se deduce de las gráficas de los tiempos de reverberación, y prueba de ello es que preguntados los alumnos al respecto, la opinión generalizada es que las cualidades acústi- cas habian variado significativamente. Por tanto, la reforma ha supuesto un avance importante en cuanto a la impresión subjetiva. No obstante, a nuestro juicio, se habría obtenido una audibilidad más adecuada si se hubiera seguido la solución que habiamos propuesto. 


\section{BIBLIOGRAFIA}

\section{Beranek, L.}

Acústica.

Ed.: Hispano Americana, S. A. Buenos Aires (1969).

Brown, A. L. et al.

"Surveying the noise exposure of classrooms". Applied Acoustics, 18, 55-67 (1985).

\section{Burgess, M. A.}

"Reverberation times in British living rooms". Applied Acoustics, 18, 369-380 (1985).

\section{Condamines, $\mathbf{R}$.}

"Qualités et défauts des salles".

Nouvelle Révue du Son, 32, 247-249 (1979).

\section{Daumal, F.}

"Nuevo método para determinar el tiempo de reverberación óptimo en auditorios".

Revista de Acústica, XVII (3 y 4), 37-43 (1986).
Daumal, F.

"Nuevo parámetro acústico-arquitectónico para sala de audiciones".

El Instalador, 209, 93-100 (1986).

\section{Daumal, F.}

"Comentarios acústicos a las reglas de oro de los tratadistas arquitectónicos".

Revista de Acústica, XVII (1 y 2), 13-19 (1986).

\section{Josse, $R$.}

La acústica en la construcción.

G. Gili, Barcelona (1975).

\section{Llopis, A. et al.}

"The effect of floor slope on speech intelligibility in rectangular rooms".

ICA 12, E 10-6.

\section{Plagnol, R.}

"Résultats de mesures acoustiques dans des salles de classe".

Cahiers du C. Sc. et T. du Batiment, 73 (620) (1965).

\section{Wernly, J. A.}

"Tiempo de reverberación en recintos prismáticos". Revista de Acústica, IX (1 y 2), 8-12 (1978).

\section{publicación del ICCET/CSIC \\ INSPECCION DE OBRAS DAÑADAS POR CORROSION DE ARMADURAS}

El presente Manual va dirigido principalmente a técnicos especializados y laboratorios que tienen que intervenir en el dictamen de la situación de deterioro de estructuras de hormigón armado dañadas por corrosión de armaduras.

Comienza con un resumen recordatorio de los factores principales a los que se pueden deber los daños prematuros por corrosión de armaduras, para seguir con algunas indicaciones de cómo se deben realizar las inspecciones, y de los ensayos y la metodología que se recomienda realizar para poder dictaminar con precisión las causas de daño.

A continuación se hacen una serie de comentarios sobre la vida residual de estructuras dañadas, sobre el riesgo de corrosión futura, el seguimiento necesario de una estructura reparada y una breve enumeración de métodos de reparación y consideraciones básicas a tener en cuenta en la recomendación de un determinado método. Se aporta una breve relación bibliográfica.

Finalmente se incluyen en forma de ficha la descripción de algunos casos de corrosión de armaduras detectados en nuestro país.

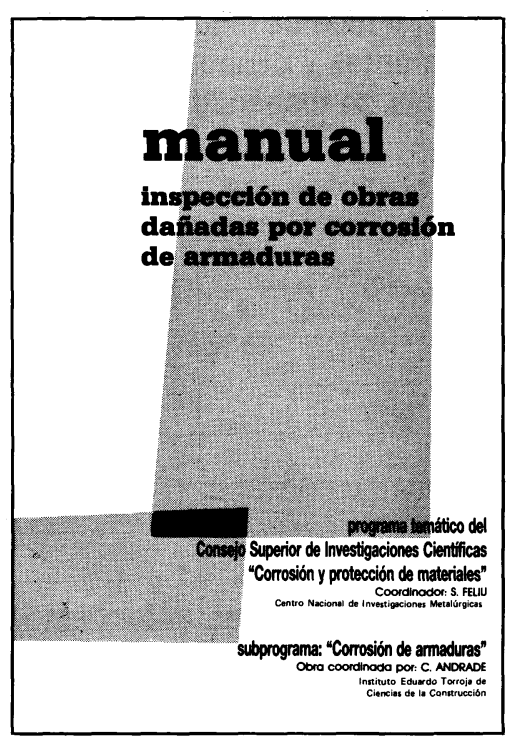

